\title{
Older Chinese Adults' Knowledge, Beliefs, Attitudes and Behaviour on Cognitive Health and Ageing: An Exploratory Study in Singapore
}

\section{Research Article}

Volume 2 Issue 1- 2021

\author{
Author Details \\ Regina Gowindah ${ }^{1}$, Ying Jie Yew ${ }^{1}$, Jeanette Hodgson ${ }^{2}$, Sarah Smith ${ }^{2}$, Jennifer Si ${ }^{1}$, Soe Pwint Phoo Mon ${ }^{1}$, Shikha Singh ${ }^{1}$ and Vince \\ Grillo $^{1 *}$ \\ ${ }^{1}$ Health Division, Kantar, Singapore \\ ${ }^{2}$ Health Division, Kantar, UK
}

*Corresponding author

Vince Grillo, General Manager, Health Division, Kantar 50 Scotts Rd, \#02-01, Singapore 228242

Article History

Received: February 04, 2021 Accepted: February 15, 2021 Published: February 16, 2021

\begin{abstract}
:
Introduction: In recent years there has been increasing strategies to delay onset or progression of dementia, and identification of its modifiable risk factors. Given the collective emphasis on dementia prevention, an in-depth exploratory study is needed to enrich previous research and provide insights on current behaviours and attitudes towards dementia prevention. We aim to investigate the knowledge, beliefs, attitude and behaviour of older Chinese adults on cognitive health and ageing to identify motivators and barriers towards activities of dementia risk reduction.
\end{abstract}

Methods \& Materials: In-depth interviews were conducted with 25 older Chinese adults between 50-75 years. The COM-B Framework from the Behavioural Change Wheel was used in the discussion guide design, and to identify themes to cover
a. knowledge on brain health;
b. perceptions of people with cognitive decline, impairment, and related conditions; and
c. habits of maintaining a healthy lifestyle.

Results: Four common insights identified were:

a. Overall understanding of dementia remained low even as the word dementia was particularly recognizable among informants;

b. Mental health was instinctively perceived to be integral to cognitive health;

c. Presence of stigma towards the at-risk population of dementia and its support services;

d. There was a basic knowledge of modifiable risk factors of dementia, but the knowledge did not always translate into preventive and health-seeking behaviour for brain health.

Conclusion: The study has adopted an innovative approach in using the COM-B Framework of Behaviour Change to unveil motivators and barriers that would influence perceptions of older Chinese adults towards cognitive health and ageing. Elements of behaviour change identified in our research can be used to support older adults and their communities to gain more awareness about dementia, reduce risks and address challenges experienced, creating a supportive community for those at risk and having dementia.

Keywords: Attitude; Behaviour; Brain Health; Cognitive Decline; Cognitive Health; Cognitive Impairment; Dementia; Healthy Ageing; Knowledge; Older adults

\section{Introduction}

Dementia is characterized by a deterioration in previously attained cognitive levels affecting activities of daily living and social functioning
[1]. In Singapore, the prevalence of dementia among adults aged 60 and above is approximately $10 \%$ [2] and as of $2014,13.1 \%$ of the resident population is aged 65 and above. This number is projected to double by 2030 [3]. Consequently, the prevalence of dementia is 
anticipated to rise. Given that dementia is a major cause of disability and dependency among older adults, it places a great burden on individuals with dementia, their caregivers, families and the wider community and society. In 2015 it was estimated that the total global societal cost of dementia was $1.1 \%$ of global Gross Domestic Product (GDP), placing a heavy economic burden on society [4]. In Singapore in the same year, an estimated $\$ \$ 2.8$ billion was spent on dementia in areas such as healthcare, medication and caregiver time costs [5]. This is expected to almost triple by 2030 [5].

Based on the World Health Organization's (WHO) Guidelines on the Risk reduction of cognitive decline and dementia in 2019, a multitude of modifiable lifestyle risk factors has been identified for cognitive impairment and dementia [6]. Recently completed large multidomain trials in Europe - FINGER, MAPT and PreDIVA [7-9] showed some promise in multidomain lifestyle interventions. Results from FINGER presented statistically significant beneficial effects of a multidomain lifestyle intervention including exercise, social activity, cognitive training and vascular and metabolic risk factor management in older adults with greater risks of cognitive decline [9]. While primary results from MAPT and PreDIVA did not show statistically significant benefits of the intervention, the intervention was effective among individuals with increased risk of dementia, having a protective effect on non-Alzheimer's dementia $[7,8]$.

Specifically, the prodromal stage of dementia or better known as Mild Cognitive Impairment (MCI) has been in the limelight in terms of research and clinical practice in the past few years [10-13]. MCI patients are recognized to be at high risk for developing progressive dementia and subsequently develop dementia [14]. Despite its tremendous burden, dementia is often seen as a normal part of ageing and not preventable $[15,16]$. Many individuals with dementia are unaware of early symptoms and present to healthcare professionals only when their condition is symptomatic or often when brain pathology is advanced. Notwithstanding the well-established relationship between modifiable risk factors with cognitive impairment and dementia, the literature on perceptions of dementia and dementia risk reduction held by older adults in Singapore has identified gaps. Findings have shown a substantial proportion of the population in Singapore lack an accurate understanding of dementia, seeing it as a part of the ageing process $[17,18]$.

Increasingly, there has been a greater focus on preventive strategies such as raising public awareness and offering support for those with dementia and their caregivers. Such efforts can help individuals to detect dementia early to better manage their condition and improve their quality of life. Given the collective emphasis on dementia prevention, an in-depth exploratory study is needed to enrich the understanding of previous research and provide insights on current behaviours and attitudes towards dementia prevention.

Past research on Chinese individuals outside of Singapore reflected how cultural beliefs were related to the normalization of dementia symptoms and their predisposition to conceal family problems [19]. Hence it is paramount to consider these cultural nuances when understanding knowledge and beliefs of cognitive impairment and dementia. This study aims to understand the knowledge, beliefs, attitude and behaviour of older Chinese adults on cognitive health and ageing. It will also identify the motivators and barriers for behaviour change to support intervention designs for dementia risk reduction, and for older Chinese adults to recognize potential risk factors for cognitive impairment and dementia. Findings from the study will highlight opportunities for intervention to delay MCI and dementia and help stakeholders such as the government and medical community to evaluate current health initiatives and unmet needs that remain to be filled for individuals and the community. Qualitative data from this study can potentially be used to inform a subsequent fullscale quantitative study involving a larger population.

\section{Methods and Materials}

\section{Design}

In-depth interviews were adopted to gain a deeper and richer understanding of older adults' perspectives on cognitive impairment and dementia, and how modifiable lifestyle factors affect cognitive impairment and dementia. In-depth interviews were preferred over semi-structured interviews and focus groups as the aim was to obtain more detailed descriptions of their experiences which would reflect their behaviours and attitudes without being influenced by other informants.

\section{Participant recruitment}

Informants were contacted using a fieldwork recruitment company through purposeful and snowball sampling to obtain their permission to participate. Only those who met all inclusion criteria and provided informed consent were eligible to participate. Informants consisted of older Chinese adults in Singapore, distributed across the lower, middle, and higher Socioeconomic Status (SES) and did not have a medical cognitive impairment diagnosis. The SES bands were determined by the latest statistics on monthly household income trends in Singapore [20] and the education level of the informants. Adults between 5075 years were identified for the study as these individuals were either approaching or have approached 65 years and above, where dementia is more common.

\section{Materials}

Given the focus of the research, the term brain health was introduced purposefully to each informant as a starting point of discussion. Previous research used brain health when referencing cognitive health as it can be easily understood by the layperson $[21,22]$. This research adopted a similar approach and cognitive health and brain health were subsequently used interchangeably. The discussion guide was informed by a review of existing literature and the COM-B framework of Behaviour Change, with an excerpt of questions illustrated in Table 1. It was structured in three sections to cover

\section{a. knowledge on brain health;}

b. perceptions of people with cognitive decline, impairment, and related conditions; and

c. habits of maintaining a healthy lifestyle.

Projective and association techniques were utilized during interviews to encourage narration and elicit intuitive responses to reveal emotional and hidden motivators. Self-completion sheets to capture lifestyle behaviours and attitudinal behaviours [23] were administered during the interviews to supplement the narrative approach. This encouraged reflective thinking on an individual basis and elicited responses that are not influenced by the presence of moderators.

Data from the interviews were analysed using a thematic analysis approach through the Behaviour Change Wheel and associated COM-B framework (shown in Figure 1). Theory provides a useful foundation for designing behavioural change interventions as interventions are often more effective when they target casual determinants of behaviour change [24]. The COM-B framework provides a deeper understanding of behaviour drawing from a single unifying theory of motivation in context that predicts what aspects of the motivational system will need to be influenced in what ways to achieve a behavioural target [25]. It identifies behavioural gaps and deficits and the potential interventions to address the gaps, through the interaction of one or more components - capability, motivation and opportunity. The framework maps interventions extracted from established theoretical approaches - Theory of Planned Behaviour, Health Belief Model and Mindspace - to systematically identify the specific behavioural components to design interventions to change behaviour [25]. 
Table 1: Discussion guide informed by the Capability, Opportunity and Motivation to Behaviour (COM-B) system, including the domains under the Theoretical Domains Framework (TDF).

\begin{tabular}{|c|c|c|c|}
\hline $\begin{array}{l}\text { COM-B } \\
\text { Construct }\end{array}$ & $\begin{array}{l}\text { COM-B Micro- } \\
\text { Construct }\end{array}$ & Domains & Eliciting questions \\
\hline \multirow[t]{2}{*}{ Capability } & Psychological & Knowledge & $\begin{array}{l}\text { What have you heard about brain health? } \\
\text { What is cognitive impairment? } \\
\text { How did the decline in cognitive function happen } \\
\text { to Person B that had unhealthy cognitive function? }\end{array}$ \\
\hline & Physical & Physical skills & $\begin{array}{l}\text { How easy is it for you to achieve <list of } \\
\text { lifestyle behaviours }>\text { ? How so? }\end{array}$ \\
\hline \multirow{2}{*}{ Opportunity } & Social & Social influences & $\begin{array}{l}\text { Please rate the extent to which you agree/ disagree: } \\
\text { I feel that it is difficult to talk to my friends and family about dementia } \\
\text { How do others in Person A/B's (with (un)healthy brain) life feel about Person A/B? }\end{array}$ \\
\hline & Physical & $\begin{array}{l}\text { Environment } \\
\text { context and } \\
\text { resources }\end{array}$ & $\begin{array}{l}\text { What are the top information sources you most frequently access for information? } \\
\text { Where do you frequently find information on health-related topics? } \\
\text { What kind of support will Person B with unhealthy brain health need? }\end{array}$ \\
\hline \multirow{5}{*}{ Motivation } & \multirow{3}{*}{ Reflective } & $\begin{array}{l}\text { Beliefs on } \\
\text { consequences }\end{array}$ & $\begin{array}{l}\text { How important are these }<\text { lifestyle activities }>\text { to brain health? } \\
\text { How will the condition change as Person B ages? }\end{array}$ \\
\hline & & $\begin{array}{l}\text { Beliefs on } \\
\text { capability / agency }\end{array}$ & $\begin{array}{l}\text { How easy is it for you to achieve }<\text { list of lifestyle behaviours }>\text { ? How so? } \\
\text { If you have been sent to advise people like Person B with unhealthy cognitive function to } \\
\text { improve their brain health, what advice would you give? }\end{array}$ \\
\hline & & Intentions / Goals & $\begin{array}{l}\text { What is important to you in your life right now? } \\
\text { What are your hopes for the future? }\end{array}$ \\
\hline & \multirow[t]{2}{*}{ Automatic } & Habits & $\begin{array}{l}\text { Which of these <lifestyle activities }>\text { have you engaged in the past year? } \\
\text { Please rate the extent to which you agree/ disagree I talk to friends and my family about } \\
\text { brain health } \\
\text { my doctor }\end{array}$ \\
\hline & & Emotion & $\begin{array}{c}\text { Please rate the extent to which you agree/ disagree I worry about dementia } \\
\text { Please rate the extent to which you agree/ disagree I am concerned about my brain health } \\
\text { declining in the future }\end{array}$ \\
\hline
\end{tabular}

Physical capability: Physical skills, strength or stamina.

Psychological capability: Knowledge or psychological skills, strength or stamina to engage in the necessary mental processes

Automatic motivation: Habits, heuristics, emotions, impulses

Reflective motivation: Reflective process involving plans (self-conscious intentions) and evaluations (beliefs about what is good and bad)

\section{Physical/ environmental opportunity:} Infrastructure, resources, time, schedule

Social opportunity: Sociocultural norms, network and relationships

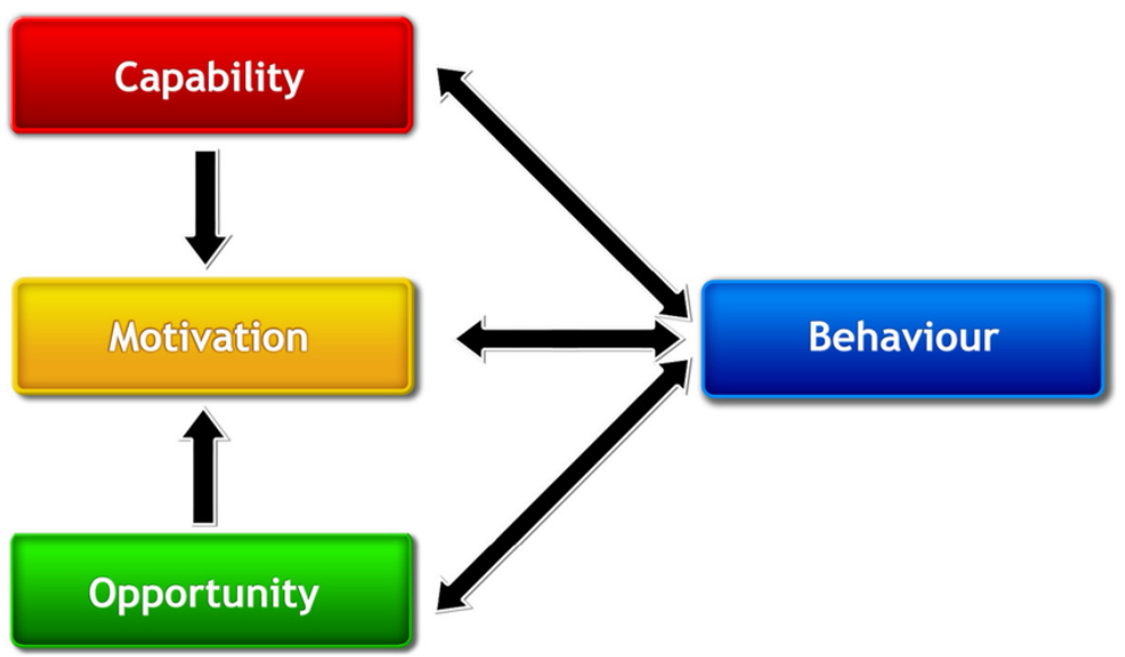

Figure 1: Components of the COM-B Framework that is part of the Behaviour Change Wheel [25].

\section{Procedure}

The protocol for this study was reviewed for exemption by Pearl Institutional Review Board (IRB) and determined to be exempt from IRB review; all informants provided informed consent after being selected for the interview. Prior to the interview informants were requested to submit a self-completion sheet in either English or Mandarin that lasted 2-3 minutes, with attitudinal statements to assess their perceptions and behaviour towards lifestyle habits and information sources with regards to brain health. Subsequently, interviews were conducted by one moderator and three researchers in the study, who ensured that the purpose of the study was clearly explained during the introduction of the interview. The moderator and researchers were competent in English and Mandarin and conducted the interviews at the convenience of the informants. All interviews were conducted virtually, one moderator or researcher with each informant and lasted for 45 minutes. Video interviews were held separately from the presence of recruiters and informants' family 
members. An incentive of $\$ 50$ was offered to informants for their participation.

\section{Data Analysis}

All interviews were recorded and transcribed. A combination of thematic and framework analysis was used, following an iterative process where the group of researchers familiarised themselves with the data, drew out themes of barriers and motivators, interpreted the data and assigned them codes. Recurrent themes were discussed with the Principal Investigator and wider team, after which final themes were determined. Finally, each theme was deductively mapped back to the COM-B framework and its domains (shown in Figure 2). A combination of thematic and framework analysis ensured that inductively identified themes were mapped back against the domains under the COM-B Framework [26]. This allowed drivers and barriers to be derived so that strategies could be identified to address the research question.

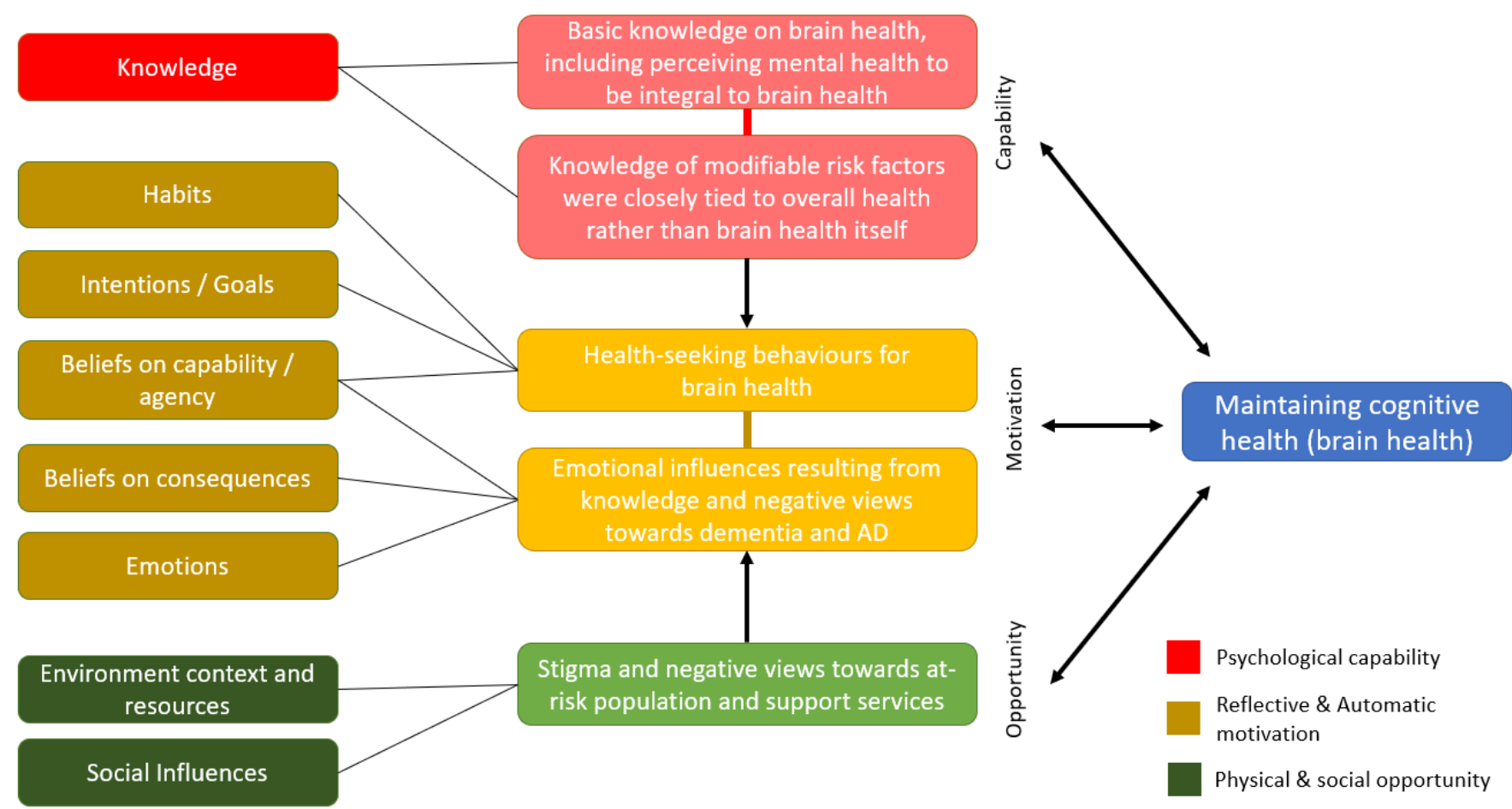

Figure 2: Themes identified inductively and mapped back against the COM-B framework and its domains.

\section{Results}

There was a range of narratives around experiences revealed by informants but also considerable consistency in the responses, such that data saturation was reached after twenty-five interviews. Using COM-B as a derivative framework, four main themes and accompanying influencers on behaviour were observed. These themes along with their respective COM-B constructs and exemplary quotes are presented and discussed in detail below.

Knowledge on Brain Health is basic but psychologically and emotionally, dementia and Alzheimer's disease evoke worry, anxiety and a sense of denial (Capability, Motivation)

Dementia and Alzheimer's Disease were value-laden terms among informants and evoked anxiety about dementia happening to them and making them a burden to their family. Throughout the interviews, this narrative resonated with various accounts of personal experiences with peers with dementia. Informants acknowledged their awareness of physical chronic conditions like cancer and diabetes was much greater in comparison to brain health because there were more touchpoints to be exposed to learn more about these conditions.

Overall, older adults were unfamiliar with brain health conditions and the differences between cognitive decline, dementia and Alzheimer's Disease. In particular, there were greater confusions among informants who spoke primarily Mandarin for the terms dementia ( 痴呆症 Chīdāi zhèng) and Alzheimer's Disease, scientifically termed 阿尔茨海默病 (Āěr cí hai mò bìng) but colloquially referred to as老 人痴呆症 (Lăorén chīdāi zhèng). The mere difference for Alzheimer's Disease colloquially was an addition of the term old person (老人
Lăorén) in front of the term dementia (痴呆症 Chīdāi zhèng). Thus, Mandarin speakers tend to mistake both conditions as being the same. In contrast, some believed that Alzheimer's Disease was a separate disorder from dementia and mistook its symptoms for Parkinson's Disease.

1. Alzheimer's Disease and Dementia are both similar to me. One of them is one category (the term old person), the other one doesn't have this category. (ID02 - Male in his 60s)

2. Alzheimer's Disease is quite like Dementia. It is also brain deterioration. The behaviour is also they tend to become forgetful, current activities also cannot be done. (ID06 - Female in her 50s)

3. Alzheimer's Disease is like Parkinson's. I think it is related to memory? This is more related to age. Somewhere in the brain is affected. I cannot tell the difference between Dementia and Alzheimer's Disease. (ID17 - Male in his 60s)

4. I see Alzheimer's Disease as the one with shaky hands and shivering. One may also urine without control and will need to wear diapers (ID19 - Female in her 70s)

5. I think cognitive impairment could relate to dementia, you may get really forgetful. I don't know what Alzheimer's Disease is. Nowadays the word dementia is so common. (ID20 - Female in her 70s)

The saliency of the recognizable symptoms of dementia, such as forgetfulness and loss of independence from loss of control of one's mental faculties, and the resulting burden to carers were the highest. Despite the fuzzy understanding for many, known aspects of these constructs invoke worry that it may happen to them one day: 
1. We do not hear of dementia and Alzheimer's so much in the public. But I do know they are forms of cognitive impairment. Because sometimes, for our age physically, mentally, when we are quite ok, we do not go in-depth in all these. We are worried that we might get this one day. (ID03 - Female in her 60s)

2. To me it is quite straightforward, it is all due to the brain functioning... whether it is healthy, deteriorating or at the worst stage (ID04 - Male in his 50s)

Responses showed that there was a lack of understanding of the basic signs and symptoms and when to watch out for them. Informants knew that these conditions are due to brain function but lack understanding of evolution or stages of brain decline. It was also observed that there may be psychological denial leading to the avoidance of seeking a deeper understanding.

Mental health was perceived to be integral to brain health and some perceive individuals with poorer mood states to have poorer cognitive health (Capability, Motivation):

Brain health was instinctively perceived to be closely knitted to mental health; the state of well-being of individuals that depends on affective states of the brain. Individuals with good brain health were seen to maintain healthy diets, active lifestyles, but also to be in a state of happiness, have better mood states and be less temperamental. These insights were raised more commonly among women:

1. I think his mood should be ok, not so temperamental. He may have healthy lifestyle, shouldn't have much of unhappiness. (ID20 - Female in her 70s)

2. If he is healthy, he cannot be a moody person in the morning. He would be more approachable, happy go lucky. The health plays an important part to his mood. If you are not healthy, wheelchairbound, you tend to shout. (ID20 - Female in her 70s)

Many were unable to separate cognitive health from mental health, and words relating to stress and emotions were most frequently articulated. Personality was also closely related to mood where happygo-lucky individuals were perceived to have better brain health

1. A person with good brain health is happy-go-lucky, he won't have any depression. If anything negative happens to him, he will take it as nothing and not be affected easily (ID10 - Female in her 60s)

A small number of informants from higher SES associated stress with cognitive health where having a good mood is important to having a clear mind. If the brain is stressed, it will work less well "ID24 - Female in her 50s". Gender differences observed in perceptions - two males felt that good brain health was not equivalent to better mood states. However, they recognised that with better cognitive health it was likely that individuals would be able to better manage their moods:

1. If you do not count any psychotic imbalance, (the person with good brain health) will be like any normal person, with a whole range of mood and emotions. He can handle it better perhaps, if he has positive mood (ID07 - Male in his 50s)

On the other hand, poor brain health would mean that one was constantly forgetful and inevitably lead to deterioration over time. This would affect Activities of Daily Living (ADL). In severe cases, it was perceived to lead to the loss of one's ability to care for oneself independently. These individuals were also deemed to be at higher risk and more susceptible to developing conditions like dementia. Generally, they felt that those with poor mental health had poorer brain health.

1. When a person is unhappy, negative, doesn't eat healthily, doesn't mix with friends and take initiatives, means they are shutting themselves away. They cannot help themselves to be happy, so there are no endorphins. The brain will deteriorate and will forget things easily. From my observations they have short attention span, and short-term memory. They are unhappy from stresses that they don't know how to disperse and keep it in the brain. Their whole-body system becomes upside down. (ID13 - Female in her 50s)

2. If you are always angry, it affects your brain health, (causes) emotional stress and anger, and affects your brain. In the long term, it leads to depression and may cause some to be abusive. (ID05 - Female in her 50s)

Furthermore, a few older females expressed prejudice towards vulnerable groups described as those with 'emotionally sensitive traits' or those from a disadvantaged background. These individuals were perceived to be suspectable to dementia and tended to have poorer cognitive health. They were associated with negative traits such as poor habits and very lazy. Economically disadvantaged individuals were felt to be more suspectable as they lack the privilege to care for their health, did not have the financial ability or lacked the social capital and knowledge to access information to maintain their brain health.

1. (Those with poorer cognitive health) Take on unhealthy activities, maybe he does not eat proper food and doesn't exercise. He is very lazy, maybe a bad person and picks quarrels. (ID19 - Female in her 70s)

2. Maybe, the person is always very depressed over something and always very worried about certain things. She may have sensitive feelings about people, always feel that people are against her. She may not be very educated and there is no way to find information on how to lead a happy, healthy lifestyle. She mixes with people who are similar in her environment. If you are with a group of healthy people that have children, they would have given her some advice. (ID22 - Female in her 70s)

The above reflects the stereotypical views of those with poorer mental health as also having poorer brain health. This points to the presence of stigma and discrimination towards people who are suspected of having poorer brain health.

\section{Stigma and negative views toward at-risk population and} support services (Opportunity, Motivation):

Despite rationalising cognitive decline to be part of the normal ageing process, most distance themselves from dementia by denying associations with it. The unfamiliarity regarding brain health deepens the stigma surrounding dementia. Narratives tend to be focused on the negative impact and burden of dementia and Alzheimer's Disease.

Through the interviews, some older informants distanced themselves, gave labels and displayed subtle discrimination towards individuals deemed less socially and cognitively active or showed signs of cognitive decline. Institutions such as Senior Activity Centres (SACs) were described as a place to keep seniors healthy, where individuals who had nothing better to do would visit when they were not able to keep themselves socially and cognitively active without the help of an institution. An informant displayed her disinterest in SACs and tried to distance herself from the idea of attending their activities:

1. I don't think I have to come to a stage to go to the senior activity centre downstairs at my void deck. I have my own activities to occupy myself. It isn't that I despise them, but it is for old people who have no activities on their own. (ID19 - Female in her 70s)

Even among the cognitively healthy, there are distinctions between those who are socially active and those who are less active and require institutional help to remain active. Among volunteers in the community, SAC activities are perceived positively but the distinction between the socially active volunteers and participants are echoed: 
1. We organize activities in the senior activity center, so that elderly will come and join the activity. Once they join the activity, they will participate, they will open up, they will say I have to do this, I have to do that, they will open up once they socialize (ID25 Male in his 70s)

As an individual's cognitive health declines, the stigma of the condition increases. When informants mention nursing homes for dementia patients, the stigma towards these individuals is much greater than that of SACs - homes are perceived as a place for those nearing the end of their lives. People with dementia are described as "very poor thing, you don't know what you are doing" (ID10 - Female in her 60s).

This is summarised by another informant on the efforts to manage individuals who are not cognitively healthy:

1. The government will put her into the nursing home. SACs will not keep you for the whole day, they are not fully responsible for your care. SACs are for normal old people, not for this stage at the end-stage. At this stage, they will not take you in. (ID19 - Female in her 70s)

Knowledge of modifiable risk factors does not always translate into preventive and health-seeking behaviour for brain health (Capability, Motivation, Opportunity):

Informants shared that there has been a greater emphasis on healthy ageing by peers, the media and community, rather than specifically maintaining brain health. They recognised the risks and contributing factors that constitute and contribute to good cognition. Good cognition was commonly associated with the terms active brain, memory and ability to remain active. While there was a good ability to list modifiable risk factors, many did not have good knowledge of how these contribute to cognitive health and tied these to the maintenance of overall health.

Maintaining a healthy lifestyle with activities such as being socially engaged, participating in cognitively stimulating activities, maintaining a healthy diet and regular exercises were more frequently mentioned. An individual's mood, maintenance of sugar levels and moderate consumption of alcohol were also specified. Causal links were observed to be more frequently drawn between brain health and being mentally stimulated, and being socially and physically active:

1. The person is using his brains a lot, thinking and analysing frequently. That's how he is using and exercising his brains every day. If you do not use your brains frequently, your brain will degenerate. If you spend the whole day idling doing nothing, you will get dementia easily. I play Sudoku very frequently, exercising my brain every day. (ID09 - Female in her 60s)

2. You need to keep yourself preoccupied to help maintain brain health. It can be playing games like Mahjong, badminton, or table tennis. Also improves your reflex action, to keep active. This helps with the cognitive function, so you know when to react at what time. (ID17 - Male in his 60s)

Besides, there were also mentions among a few informants that physical aspects of the brain would affect brain health such as brain injuries:

1. Our limbs are getting old. If we have a fall and fall on our brains, it can trigger damage to the brain. This happened to my grandmother-in-law. She fell which caused a blood clot in her brain. The function of the whole body becomes weaker. (ID15 Female in her 50s)

All informants perceived age as a main risk factor affecting brain health and many believed that cognitive decline is a normal part of ageing and cannot be prevented at later ages. Few informants were aware that poorer cognitive health can occur among younger age groups. Some recognised that many could not distinguish between dementia and characteristics of normal ageing:

1. When (we grow older) we may do things a little slower, response is slower, not as fast as our younger times. So slow and then sometimes we might miss out certain things. But whether it is a normal way of growing old or what... the word dementia and growing old, many people don't know the difference. We take it for granted that they (growing old and dementia) are the same (ID03 - Female in her 60s)

There were mixed perceptions on when an individual should be concerned about cognitive decline, ranging from the early 50 s to the 60s. Responses were based on the life stage where one approaches retirement rather than age itself. Retirement was most salient as a stage of reduced cognitive and social activities. Males made more obvious links between cognitive decline and retirement, although a good number of interviewed females were still working.

1. Slowing of brain probably happens at age 60 at retirement age. Probably the time we become slower in our activities, not engaged in much thinking work. (ID04 - Male in his 50s)

2. I know as we grow older, the brain cells die. To make the brain cells work, you must work, make your brain work like thinking have a bit of pressure, tell people off, go for meetings. I heard from people when you retire, you do not think. (ID01 - Male in his 60s)

Fears of a deteriorating brain, decreasing memory loss and developing dementia were the main motivators to engage in cognitively stimulating activities for a few informants:

1. I have been playing games. Sometimes if I go to the residential committee centre I will play Rummy-O, a game similar to Mahjong. I started playing that to prevent dementia (ID22 Female in her 70s).

Informants were able to engage in cognitively stimulating activities but many did not pursue these purposefully for brain health. Motivations were often a result of intentions to maintain the physical or the overall health of the body. The intention to engage activities to maintain cognitive health was less apparent for the remaining modifiable lifestyle risk factors including exercising, being socially engaged, maintaining blood pressure and sugar levels, moderate consumption of alcohol and avoiding smoking.

Tied in with preventive measures, is the intention and ability to seek medical help in the event of a persistent cognitive decline. Informants highlighted the lack of knowledge to seek active help. Most did not know when or from whom to seek help and identified the lack of a "trigger point" for brain checks, unlike other chronic diseases that can be detected through health check-ups. Some males were better informed and knew help could be sought from a psychiatrist or neurologist in an event of a persistent decline in cognitive activity. However, when questioned on their intent to visit the doctor in an event of suspected cognitive decline, some informants mentioned they would only be ready to speak to a healthcare professional on brain health when there are obvious symptoms:

1. Now I do not need to talk to doctor. I am so healthy, so I have no need to talk to (a) doctor. (ID19 - Female in her 70s)

The above reflects the rationale that as long one is in good health, there is no need to actively seek information or consultation about dementia and related conditions. Throughout our interviews, the majority claim to be comfortable talking to their peers about dementia; yet, inputs from the self-completion sheets showed that they would not initiate a conversation with their doctors about their brain health. 


\section{Discussion}

The present study explored the perceptions of older Chinese adults on cognitive health and ageing. Findings show that overall understanding of dementia remained low even as the word dementia was particularly recognizable among informants. Mental health was instinctively perceived to be integral to cognitive health, and there was some stigma towards the at-risk population of dementia and its support services. While there was a basic knowledge of modifiable risk factors of dementia, the knowledge did not always translate into preventive and health-seeking behaviour for brain health.

This study is the first of its kind in Singapore to lend a voice to individuals of the general public to understand their perceptions on cognition and ageing, as previous studies on perceptions of cognitive health in Singapore were mainly quantitative or stakeholder focused (carers, healthcare professionals, policymakers) [17,27-30]. Results are congruent with previous research demonstrating the lack of knowledge of dementia among the general public $[17,27,31]$. However, our research went further to probe on older adults' lack of knowledge of the progression of dementia and its prodromal stages to uncover inherent cognitive biases and stigma hindering adoption of support services and help-seeking behaviour.

Insights align with earlier research identifying general practitioners as the main source of help recommended by the general public for dementia rather than psychiatrists or psychologists [32]. Routine screening for dementia in Singapore is only recommended when there is a persistent decline in cognitive activities such as those relating to memory [33]. The first points of contact advised were gerontologists or specialists at memory clinics within public hospitals, which were not mentioned by any informants. Findings show low awareness of what specifically constitutes persistent decline in cognitive activities that would lead to dementia among the general population. The lines are blurred between what is viewed as a natural part of ageing and a progression that requires medical attention. Furthermore, the overlap with mental health has added to the confusion and deepened the stigma to speak to healthcare professionals about their health, as conversations on severe illnesses are often construed to the Chinese as inauspicious or bad luck [34].

Although not explicitly mentioned, both anticipated and internalized stigma [35] were reflected in interviews and heavily influenced mental health treatment. This study showed this is not confined to treatment-seeking and included intended approaches in managing cognitive decline and dementia. Negative sentiments demonstrated in interviews towards institutionalized individuals, reveals anticipated stigma - the label of being not normal are put on those with declining cognitive health.

Concurrently, self-stigmatization plays a role in contributing to the negative perceptions when individuals endorse the negative beliefs and feelings associated with cognitive decline, dementia and Alzheimer's Disease. Previous research has shown that there is general acceptance that cognitive decline is a natural part of ageing [17], which ultimately impacts health-seeking behaviour. Many informants interviewed mentioned they would only be ready to speak to a healthcare professional on brain health when there are obvious symptoms. These insights expand on quantitative findings that persons with dementia and their caregivers experience shame and feel less competent [31] and that the lay public would also feel a sense of shame if they had dementia [17].

The perception towards an individual is seen to be closely tied to the nature of the help that one receives. Singapore as a non-welfare state has built its social net on three main principles - self-reliance, family as the first line of support and the Many Helping Hands approach [36]. The society encourages individuals to be self-reliant, before turning to the family as the first line of support. Only when all else fails where one falls through the social nets and still requires assistance, does one receive assistance from institutions in the community. Thus, the stigma towards mental health and help-seeking is deeply entrenched in these societal structures - receiving services from institutions is perceived as being needy and not having the first line of support from the family. As a country that continues to uphold Confucian values, viewing the family unit as the basic building block of society, the shame is not just of having poorer brain health and being dependent on peers but also not having the fundamental family support, culturally viewed as of utmost importance [37].

Importantly the healthcare system needs to consider developing pathways for individuals suspected of persistent cognitive decline to seek help especially for prodromal and mild stages, where there is a greater chance for delaying onset or progression. On the other end of the spectrum, dementia needs to be highlighted not as a natural path of ageing, but a condition that can be delayed or prevented to avoid decline. A deeper understanding of the perceptions of the healthcare and social services for eldercare and its effectiveness would be useful. The issue of stigma is fundamental in influencing behaviour change; yet, most challenging as it involves preconceived notions of what constitutes dementia. Concerted efforts to engage the general public to dispel misconceptions can be implemented to support and guide the needs of a population group at risk or with poor cognitive health. This empowers the general public to create their own narrative of ageing and cognitive health to focus on optimism and support for those living with the condition. Efforts can be sustained and scaled up to incorporate elements of behaviour change identified in our research to support older adults and their communities to gain more awareness about dementia, reduce risks and address challenges experienced, creating a supportive community for those at risk and having dementia.

The study has some limitations. Due to the sample selection during recruitment, informants who are generally better informed are more likely to participate and be interested in the research. Hence the possibility of selection bias; the results may not be reflective of the entire Chinese population in Singapore. There may be heterogeneity among informants in their degree of interaction with individuals with dementia, depending on whether they are a caregiver, family, friend or acquaintance or know of people with dementia.

\section{Conclusion}

The study has adopted an innovative approach in using the COM-B Framework and its domains to unveil motivators and barriers that would influence perceptions of older Chinese adults towards cognitive health and ageing. Additionally, the maintenance of modifiable lifestyle behaviours to maintain cognitive health was also explored. By utilising the COM-B Framework and projective techniques, the researchers ensured that the study was person-centered rather than researcher-led while retaining its theoretical rigour. With a better awareness of current knowledge and attitudes and an understanding of the environment towards cognitive conditions such as dementia, community and government initiatives can incorporate a multifaceted approach in reducing stigma and discrimination towards dementia and related support services.

\section{Acknowledgement}

The authors acknowledge the support provided by the Society of Behavioural Health, Singapore who acted as advisors in developing the manuscript. We would also like to thank all informants for their cooperation.

\section{Statement of Ethics}

The protocol for this study was reviewed for exemption by Pearl 
Institutional Review Board (IRB) and determined to be exempt from IRB review in accordance with FDA 21 CFR 56.104 and 45CFR46.104. All informants provided informed consent to participate.

\section{Funding Sources}

This study was funded by Health Division, Kantar, Singapore for the conduct of the study and development of the manuscript.

\section{Author Contributions}

V.G., R.G. and Y.J.Y. led and all authors contributed in the design of the study and the analysis. R.G., Y.J.Y., J.H., S.S., and J.S. were involved in interpreting the data. R.G., Y.J.Y. and J.S. reviewed the literature and conducted the interviews. R.G. and Y.J.Y. led the manuscript writing and all authors contributed in the writing of manuscript, provided input at the writing stage and the final draft. All authors read and approved the final version of the manuscript.

\section{References}

1. World Health Organization (2020) International statistical classification of diseases and related health problems: $11^{\text {th }}$ Revision (ICD-11).

2. Subramaniam M, Chong SA, Vaingankar JA, Abdin E, Chua BY, Chua HC, et al. (2015) Prevalence of Dementia in People Aged 60 Years and Above: Results from the WiSE Study. J Alzheimers Dis. 45(4): $1127-$ 1138.

3. Department of Statistics Singapore (2015) Population Brief.

4. World Health Organization (2020) Dementia.

5. Woo LL, Thompson CL, Magadi H (2017) Monetary cost of family caregiving for people with dementia in Singapore. Arch Gerontol Geriatr. 71: 59-65.

6. World Health Organization (2019) Risk reduction of cognitive decline and dementia: WHO guidelines.

7. Andrieu S, Guyonnet S, Coley N, Cantet C, Bonnefoy M, et al. (2017) Effect of long-term omega 3 polyunsaturated fatty acid supplementation with or without multidomain intervention on cognitive function in elderly adults with memory complaints (MAPT): a randomised, placebo-controlled trial. Lancet Neurol. 16(5): 377-389.

8. Moll van Charante EP, Richard E, Eurelings LS, van Dalen J-W, Ligthart SA, et al. (2016) Effectiveness of a 6-year multidomain vascular care intervention to prevent dementia (preDIVA): a cluster-randomised controlled trial. Lancet Lond Engl. 388(10046): 797-805.

9. Ngandu T, Lehtisalo J, Solomon A, Levälahti E, Ahtiluoto S, et al. (2015) A 2 year multidomain intervention of diet, exercise, cognitive training, and vascular risk monitoring versus control to prevent cognitive decline in at-risk elderly people (FINGER): a randomised controlled trial. Lancet Lond Engl. 385(9984): 2255-2263.

10. Abbate C, Trimarchi PD, Inglese S, Gallucci A, Tomasini E, et al. (2020) The Two-Step Strategy Could Be Inadequate and Counteracting to Diagnose Prodromal Dementia or Mild Cognitive Impairment. Front Aging Neurosci.12.

11. Petersen RC (2004) Mild cognitive impairment as a diagnostic entity. J Intern Med. 256(3): 183-194.

12. Petersen RC, Smith GE, Waring SC, Ivnik RJ, Tangalos EG, et al. (1999) Mild cognitive impairment: clinical characterization and outcome. Arch Neurol. 56(3): 303-308.

13. Vos SJB, Verhey F, Frölich L, Kornhuber J, Wiltfang J, et al. (2015) Prevalence and prognosis of Alzheimer's disease at the mild cognitive impairment stage. Brain J Neurol. 138(Pt 5): 1327-1338.

14. Alcove Project (2013) The European Joint Action on Dementia. Report on the Benefits and the Risks of Dementia Diagnosis. Alzheimer Cooperative Valuation.

15. Clare L, Goater T, Woods B (2006) Illness representations in early-stage dementia: a preliminary investigation. Int J Geriatr Psychiatry. 21(8): 761-777.
16. Flicker L (2009) Life style interventions to reduce the risk of dementia. Maturitas. 63(4): 319-322.

17. Tan WJ, Hong S-I, Luo N, Lo TJ, Yap P (2012) The Lay Public's Understanding and Perception of Dementia in a Developed Asian Nation. Dement Geriatr Cogn Disord EXTRA. 2(1): 433-444.

18. Livingston G, Sommerlad A, Orgeta V, Costafreda SG, Huntley J, et al. (2017) Dementia prevention, intervention, and care. Lancet Lond Engl. 390(10113): 2673-2734

19. Prince M10/66 Dementia Research Group (2004) Care arrangements for people with dementia in developing countries. Int J Geriatr Psychiatry. 19(2):170-177.

20. Department of Statistics Singapore (2019) Key Household Income Trends.

21. Laditka JN, Beard RL, Bryant LL, Fetterman D, Hunter R, et al. (2009) Promoting cognitive health: a formative research collaboration of the healthy aging research network. The Gerontologist. 49(Suppl 1): S12-S17.

22. Laditka SB, Corwin SJ, Laditka JN, Liu R, Tseng W, et al. (2009) Attitudes about aging well among a diverse group of older Americans: implications for promoting cognitive health. The Gerontologist. 49 (Suppl 1): S30-S39.

23. Kraus L, Augustin R (2001) Measuring alcohol consumption and alcohol-related problems: comparison of responses from selfadministered questionnaires and telephone interviews. Addict Abingdon Engl. 96(3): 459-471.

24. Michie S, Johnston M, Francis J, Hardeman W, Eccles M (2008) From Theory to Intervention: Mapping Theoretically Derived Behavioural Determinants to Behaviour Change Techniques. Appl Psychol. 57(4): $660-680$.

25. Michie S, van Stralen MM, West R (2011) The behaviour change wheel: a new method for characterising and designing behaviour change interventions. Implement Sci IS. 6: 42.

26. Gale NK, Heath G, Cameron E, Rashid S, Redwood S (2013) Using the framework method for the analysis of qualitative data in multidisciplinary health research. BMC Med Res Methodol. 13(1): 1-8.

27. Setia M, Islam AM, Thompson JP, Matchar DB (2011) Stakeholders' perspective on issues and challenges associated with care and treatment of aging-related cognitive impairment disorders in Singapore. Int Psychogeriatr. 23(9): 1421-1432.

28. Tan GTH, Yuan Q, Devi F, Wang P, Ng LL, et al. (2020) Dementia knowledge and its demographic correlates amongst informal dementia caregivers in Singapore. Aging Ment Health. Pp:1-9.

29. Alzheimer's Disease Association (2015) Towards A Dementia-Friendly Singapore" Focus Group Discussions Report.

30. Zhang F, Cheng S-T (2020) Does exposure to information about dementia change stigma? An experimental study. Aging Ment Health. 24(7): 1161-1166.

31. Alzheimer's Disease Association (2019) 3 in 4 persons with dementia feel rejection and loneliness, national survey on dementia finds Alzheimer's Disease Association, Singapore.

32. Picco L, Abdin E, Chong SA, Pang S, Vaingankar JA, et al. (2016) Beliefs about help seeking for mental disorders: Findings from a mental health literacy study in Singapore. Psychiatr Serv. 67(11): 1246-1253.

33. Agency for Integrated Care (2020) Mind Matters: A Resource Directory on Community Mental Health.

34. Elliott KS, Di Minno M, Lam D, Tu AM (1996) Working with Chinese families in the context of dementia. Ethn Dement, pp: 89-108.

35. Fox AB, Smith BN, Vogt D (2018) How and when does mental illness stigma impact treatment seeking? Longitudinal examination of relationships between anticipated and internalized stigma, symptom severity, and mental health service use. Psychiatry Res. 268: 15-20. 
36. Lim XH (2007) Security with self-reliance: The argument for the Singapore model. Ethos J Soc Psychol Anthropol (3): 35-39.
37. Ministry of Social and Family Development (2006) Report of the Committee on Ageing Issues. Ministry of Social and Family Development. 